\title{
The X-ray detection performance of polycrystalline CVD diamond with pulsed laser deposited carbon electrodes
}

\author{
Mohamed A.E. Abdel-Rahman ${ }^{*}$, , Annika Lohstroh $^{1}$, Imalka Jayawardena ${ }^{2}$ and Simon J. Henley ${ }^{2}$ \\ ${ }^{1}$ Department of Physics, University of Surrey, Guildford, Surrey GU2 7XH, United Kingdom \\ ${ }^{2}$ Advanced Technology Institute, University of Surrey, Guildford, Surrey, United Kingdom
}

\begin{abstract}
The deposition of amorphous carbon electrical contacts on a diamond radiation detector by Pulsed Laser Deposition (PLD) is introduced as a novel technique for producing tissue equivalent x-ray dosimeters. Three devices were fabricated with the following electrical contacts: pure amorphous carbon (labelled Poly-C), amorphous carbon mixed with Nickel (PLD) (labelled Poly-C/Ni) and conventional sputtered Pt (labelled Poly-Pt). To examine the performance of PLD carbon as a contact, a set of $\mathrm{x}$-ray detection characteristics was studied and compared to those of Poly-Pt. This investigation includes current-voltage characteristics, linearity and dose rate dependence, sensitivity and specific sensitivity, photoconductive gain, stability, reproducibility and time response (rise and fall-off times). The experimental results suggest that Poly-C/Ni is suitable for an $\mathrm{x}$ ray dosimeter. It shows a high signal to noise ratio $(\mathrm{SNR})$ of $\sim 3300$, approximately linear relationship between the photocurrent and the dose rate and a sensitivity of $65 \mathrm{nC} / \mathrm{Gy}$. In addition the current signal is stable and reproducible (within $0.26 \%$ ) and the rise and fall-off times are less than 1.1 and 0.4 seconds, respectively.
\end{abstract}

Keywords: CVD diamond, radiation detector, dosimetric characteristics, carbon electrode, PLD

\section{Introduction}

The corrosion resistance and the radiation hardness of diamond allow it to be used for measuring radiation in difficult circumstances / harsh environments such as in nuclear waste monitoring [1]. The strong atomic bonding in the crystal structure is an advantage in diamond, which gives the material the ability to measure high intensity ionizing radiation with a long device life time.

Diamond is regarded as a suitable material for radiation detection purposes when used as a solid-state ionization chamber. One of its advantages is its near tissue equivalence [1-6] where its atomic number is comparable to that of human tissue ( $\mathrm{Z}=6$ for diamond and $\mathrm{Z} \approx 7.5$ for human tissues). Thus the energy absorbed by diamond is similar to that absorbed by human tissue, which reduces the need for correction mechanisms as with other semiconductor materials [6]. Its high bandgap (5.5 eV) [1, 3, 7-9] ensures a low dark current and hence a low background noise. It has a low sensitivity to the visible and IR parts of the spectrum, which is sometimes termed visible blindness [10], making it optimum for use in deep UV/photo detection. It also has a high electron and hole mobility [2, 3, 7, 8], which permits a fast time response of the signal.

It is preferable to use electrical contacts with low thickness and low atomic number in medical applications. For this reason, carbon as an electrical contact is an ideal choice. PLD of amorphous carbon and carbon/metal mixtures allow us to control the electrical properties of the electrode layer by the variation of deposition parameters. Amorphous carbon films (containing a high proportion of $\mathrm{sp}^{3}$ bonds) are called diamond-like carbon (DLC) as they have similar properties to diamond. Of particular interest in this context are radiation hardness, tissue equivalence, non-toxicity and chemical inertness [2, 3]. Therefore the development of DLC as electrodes films will help applications in medical x-ray dosimetry.

\footnotetext{
* Corresponding author: e-mail m.abdel-rahman@surrey.ac.uk, Phone: 0020122 2431122, +44 (0) 1483686781
} 
In this work, we compare the dosimetric characteristics of three polycrystalline diamond devices. Two of these devices are contacted with Carbon and a mixed $\mathrm{C} / \mathrm{Ni}$ film by pulsed laser ablation of a graphite and a mixed graphite/Nickel target respectively. The third device is metalized by conventional sputtering of Platinum for comparison.

The deposition by a laser helps the formation of a layer of carbon with a high quality and a low percentage of $\mathrm{sp}^{2}$ hybridization [3], which in turn reduces the conductivity of the material and consequently reduces the dark current. The quality of this film depends on both the ratio of $\mathrm{sp}^{3} / \mathrm{sp}^{2}$ bonded carbon in the film and also on many preparation parameters such as the laser fluence, number of laser shots used, pulse duration, and nature of the graphite target [4].

\section{Sample preparation}

\subsection{Device Fabrication}

The following section describes the equipment and process involved in Diamond like Carbon (DLC) deposition onto the polycrystalline diamond crystals using PLD as shown in figure (1). The starting materials were thermal grade polycrystalline diamond films, (TM180) obtained from Element Six Ltd in 2010 in one batch with polished surfaces. The dimensions of these materials are $10.5 \times 10.5 \times 0.5 \mathrm{~mm}^{3}$, with a thermal conductivity $>1800 \mathrm{Wm}^{-1} \mathrm{~K}^{-1}$. Prior to the electrode deposition, all three polycrystalline samples were identical. Each diamond crystal was then chemically treated for oxidation before DLC deposition to improve the surface properties and to make sure, an oxygen terminated surface with low dark current (high resistivity) was created. For the oxidation of the sample 5 grams of potassium nitrate $\left(\mathrm{KNO}_{3}\right)$ were mixed with $20 \mathrm{ml}$ of concentrated sulphuric acid $\left(\mathrm{H}_{2} \mathrm{SO}_{4}\right)$ and heated to a temperature of $300{ }^{\circ} \mathrm{C}$ [11]. Each crystal was then submerged in the boiling solution for 5 minutes; the crystal was then washed with de-ionized water followed by acetone. This was then followed by washing in isopropanol and at last another wash by using de-ionized water.

As illustrated in Fig. 1, multiple sample holders and a rotating target holder are housed inside a vacuum chamber (typically $6 \times 10^{-6}$ Torr). A $25 \mathrm{~ns}$ pulsed $\mathrm{KrF}$ excimer laser (Lambda- Physik LPX 210i) operating at $248 \mathrm{~nm}$ is focused through a quartz window onto the target to evaporate the material and form a plasma plume which then deposits a thin film on the surface of the sample. The deposited material is amorphous in nature. By ablating an $\mathrm{sp}^{2}$ rich target with short laser pulses a plasma plume with energetic species (ions, small carbon clusters) are formed. Due to the lack of a background gas, the carbon deposited on the substrate is also highly energetic in nature and leads to the formation of an amorphous film that is more Diamond like in nature [12].

The focused laser beam falls on the carbon target surface (which is $6 \mathrm{~cm}$ away from the samples) with an angle of $45^{\circ}$ with respect to the normal of the target surface. Before any deposition the target is cleaned for 10 minutes while rotating with a speed of $40 \mathrm{rpm}$ to remove any contamination present on the top layer of the target surface. During the deposition process, the target is also rotated at the same speed. Deposition of each film was carried out using 2500 laser shots.

In order to determine the optimum fluence required for the carbon to adhere to the diamond sample and to give a

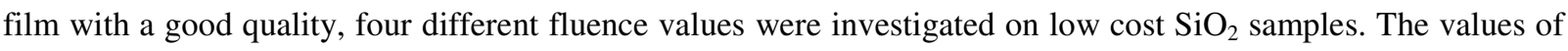
the fluence used were $3,4,5$ and $6 \mathrm{~J} / \mathrm{cm}^{2}$. It was found that the increase of the fluence results in the increase of stress which results in poor adhesion (adherence) of the deposited layer. A fluence of $4 \mathrm{~J} / \mathrm{cm}^{2}$ (focused to a spot size of $2 \times 0.5 \mathrm{~mm}^{2}$ ) was found to be the optimum and was achieved using $40 \mathrm{~mJ}$ of energy per pulse with a repetition rate of $10 \mathrm{~Hz}$. 


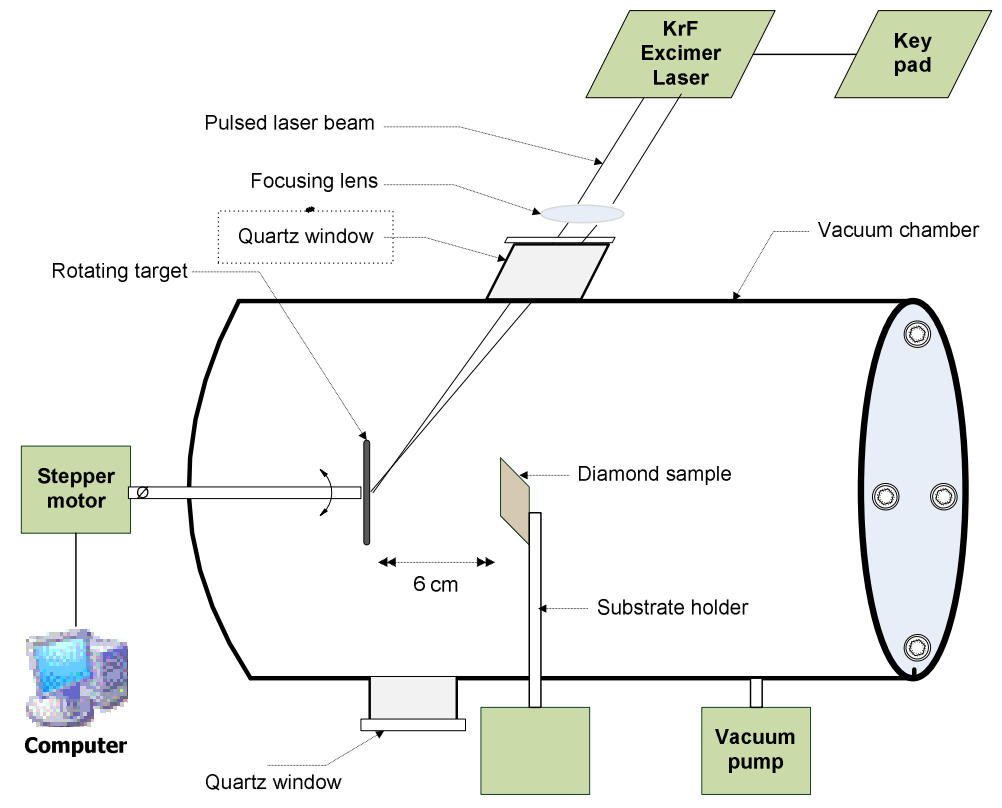

Figure (1): Schematic diagram of the thin film deposition on the polycrystalline diamond using the Pulsed Laser Deposition (PLD) technique.

A shadow mask was placed on the samples that incorporated three circles of diameters 2,4 and $5 \mathrm{~mm}$ creating three devices on each crystal. Two different targets were used for the fabrication of the devices by this method. The first was a pyrolytic graphite target (Kurt J. Lesker, with a purity of $99.99 \%$ ) and the second target used was C/Ni: 80:20 (at. \%). The third device was fabricated by conventional sputtering of Pt (using an Edwards K575XD Turbo Pumped High Resolution Sputter Coater). The three polycrystalline diamond detectors fabricated were labelled Poly-C (with $25 \mathrm{~nm} \mathrm{C}$ contacts), Poly-C/Ni (with $80 \mathrm{~nm} \mathrm{C/Ni} \mathrm{contacts)} \mathrm{and} \mathrm{Poly-Pt} \mathrm{(with} 100 \mathrm{~nm} \mathrm{Pt}$ contacts) accordingly. After contact deposition the devices were annealed at $600{ }^{\circ} \mathrm{C}$ for 10 minutes, to increase electron delocalization through graphitization leading to better electrical properties [12]. This was achieved using a Lenton low pressure furnace operated with $100 \mathrm{sccm}$ of Helium. The ramp rate of the heating oven is in the 15$20^{\circ} \mathrm{C} / \mathrm{min}$ range.

The three devices were mounted on printed circuit board (PCB), using a small dot of gold paste on the centre of the three deposited circles. Three gold wires (thickness of $24 \mu \mathrm{m}$ ) were attached to the three circles on the top using Conductive Silver Epoxy. The other end of the gold wires were connected to their corresponding copper pads on the PCB using silver paint as shown in figure (2). The pads are connected with standard electrical wires connected to a BNC bulkhead connector.

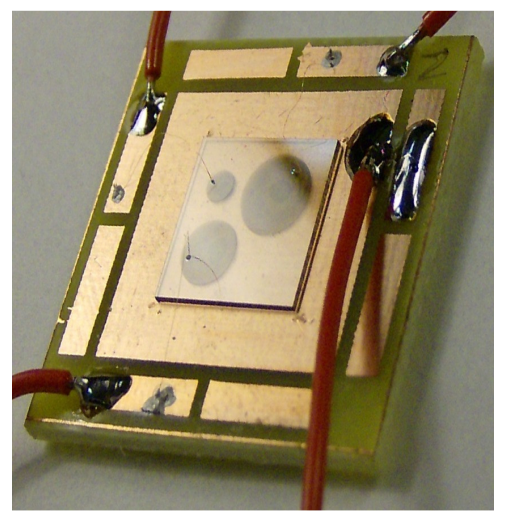

Figure (2): Photograph of the Poly-C/Ni diamond detector. 
For the purpose of the following experiments only the metal contact of size of $4 \mathrm{~mm}$ diameter was used when performing any measurements. It should be noted that the three contacts on each of three samples was tested and consistent data observed.

\subsection{Characterisation of the PLD layers}

\section{Raman spectroscopy}

The structure of the deposited carbon film on polycrystalline diamond was characterized before and after annealing using Raman spectroscopy. The Raman spectroscopy was carried out using a Renishaw MicroRaman system at a wavelength of $514.5 \mathrm{~nm}$.

The result shows prior to annealing, the existence of a $\mathrm{G}$ peak as seen in figure (3.a); it means that the carbon film has $\mathrm{sp}^{2}$ bonded atoms in the film. Additionally, the weak $\mathrm{D}$ peak indicates very little disordered hexagonal $\mathrm{sp}^{2}$ content in the film [7]. The D position, G position and the G peak FWHM are indicated in table 1. This peak shape is consistent with the deposition of high $\mathrm{sp}^{3}$ DLC [6]. The $\mathrm{sp}^{3}$ fractions for the pure carbon films come to around $70 \%$ [13]. For the $\mathrm{C}: \mathrm{Ni}$ films the $\% \mathrm{sp}^{3}$ is unknown and possibly difficult to evaluate without careful consideration, due to the large metal fraction in these films.

\begin{tabular}{c|cc|c}
\hline & D pos $\left(\mathbf{c m}^{-\mathbf{1}}\right)$ & G pos $\left(\mathbf{c m}^{-\mathbf{1}}\right)$ & G FWHM \\
\hline Before annealing & 1350 & 1550 & 100 \\
\hline After annealing & 1385 & 1580 & 70 \\
\hline
\end{tabular}

Table (1): G and D peaks of the Poly-C before and after annealing

However, after annealing $\mathrm{sp}^{2}$ bonded chains become graphitic clusters. The D peak intensity increases as the D peak indicates defects in $\mathrm{sp}^{2}$ hexagons (i.e. edges of $\mathrm{sp}^{2}$ hexagons).
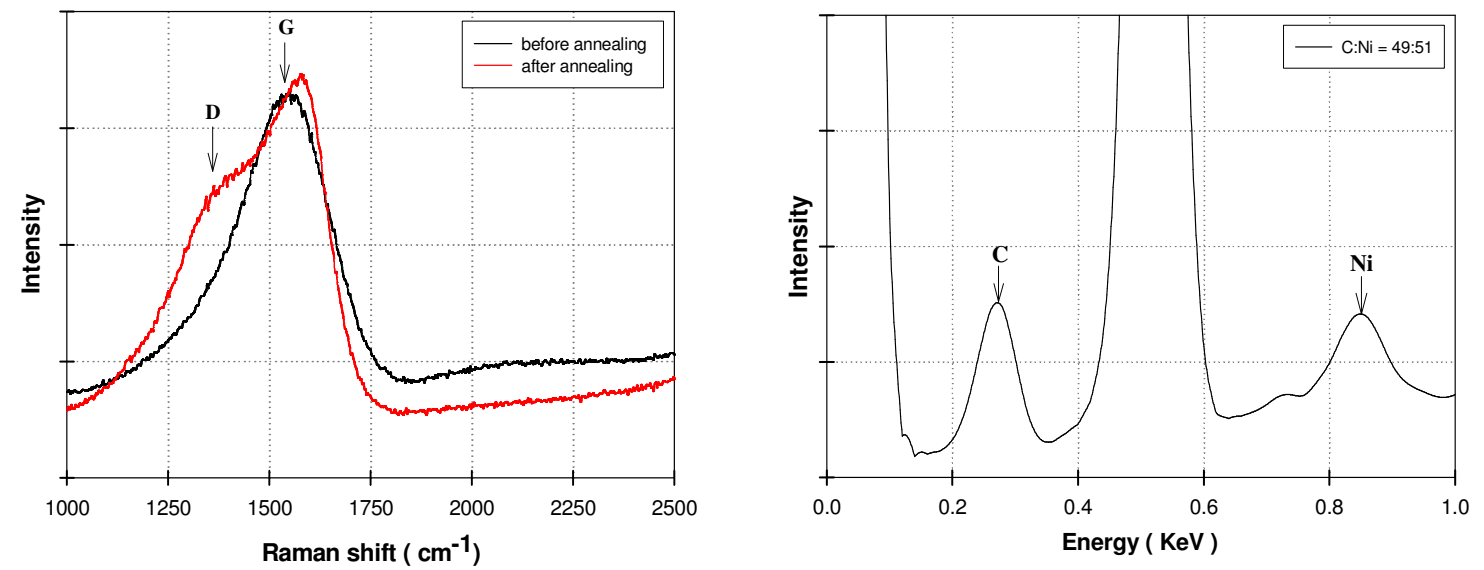

Figure (3): (a) Raman spectroscopy of the Poly-C before and after annealing (b) Energy Dispersive X-ray (EDX) of the $\mathrm{SiO}_{2}$ - $\mathrm{C} / \mathrm{Ni}$ device.

An Energy Dispersive X-ray (EDX) measurement was performed to check the Ni fraction in the deposited film as shown in figure (3.b). The Carbon/Nickel ratio was determined (as atomic percentages) from films deposited on a $\mathrm{SiO}_{2} / \mathrm{Si}$ substrate with the aid of an Oxford INCA Penta FETx3 EDX system in combination with a FEI Quanta 200F scanning electron microscope. In order to quantify the results, an EDX spectrum of the target which had a known composition of 49:51 (atomic \%) of C: Ni was also taken. Calculation of the Carbon and Nickel percentages was done using Gaussian fits. 


\section{Electrical characterisation and x-ray measurements}

\subsection{I-V characteristics}

The current-voltage characteristic of the three polycrystalline diamond devices was investigated in dark conditions and under X-ray irradiation as shown in figure (4.a-c). The broad beam X-ray irradiation of the three devices was carried out in air using a $50 \mathrm{kVp}$ X-ray tube with a molybdenum reflection target (Oxford instruments XF50 11). Different dose rates were adjusted by varying the anode current. The quoted values correspond to the air kerma near the sample position calibrated by a $0.6 \mathrm{~cm}^{3}$ ionization chamber of model number NE2571A; this gives a crude approximation of the energy deposited in the samples neglecting differences due to air ionization, scattering, and absorption effects.

The bias voltage is applied through the top contact and varied from $-200 \mathrm{~V}$ to 0 then from 0 to $+200 \mathrm{~V}$ with voltage steps of $2 \mathrm{~V}$. All current measurements were performed using a Keithley 487 Pico ampere meter which was adjusted to record the current after 30 seconds stabilisation time, in order to measure the equilibrium current. The three devices were pre-irradiated with an x-ray dose of $\sim 10$ Gy before any measurements were taken.
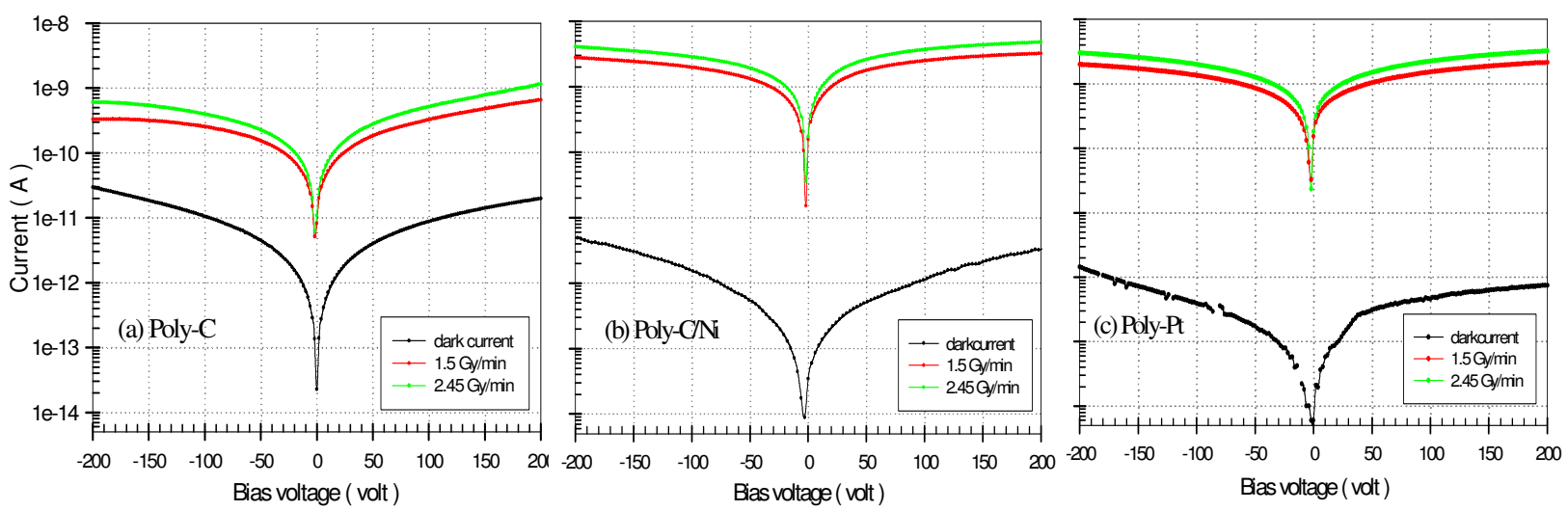

Figure (4): I-V characteristics of the three diamond samples (a) Poly-C (b) Poly-C/Ni (c) Poly-Pt both in dark conditions and under different dose rates of 1.5 and $2.45 \mathrm{~Gy} / \mathrm{min}$.

It is clear from figure (4) that the dark currents are very low (below 25 pA between $-200 \mathrm{~V}$ and $+200 \mathrm{~V}$ ) which implies that these devices have a high resistance $\left(\sim 1.1 \times 10^{14} \Omega\right.$ - deduced from the dark current at $\left.+100 \mathrm{~V}\right)$. The highest dark current is observed in Poly-C which could be attributed to the presence of defects, either presented in the bulk material or at the contact-material interface. We do not believe that the high dark current comes from defects in the bulk material as all three polycrystalline materials have been manufactured to the same specifications. The above results may indicate that the defects may be introduced in the carbon-diamond interface during the deposition of carbon onto the polycrystalline sample. These defects create energy levels within the bandgap of the device. These defect levels increase the transition probability of electrons from valance band (VB) to conduction band $(\mathrm{CB})$ via thermal excitation. A valence band electron can be thermally excited readily to a defect level and subsequently to the CB there by generating a free electron-hole pair. This kind of defect assisted in the thermal generation of electron hole pairs, which increase the magnitude of the dark current. Another possible explanation could be the carbon deposition process changed the $\mathrm{sp}^{3}$ bonds between the carbon atoms in the diamond surface, below the contacted area, into a $\mathrm{sp}^{2}$ bonds which is more stable at room temperature. As such this surface area could be changed to a graphite film, which has a high conductivity leading to a reduction in the surface resistance after annealing which could contribute to the electrical conduction of the dark current. 
In all three devices slight asymmetry is observed with bias showing consistency with those results reported by Benedetto et.al. [8] who attributed it to the difference in junction barriers at the electrode diamond interfaces. This is assumed to be caused by limited reproducibility of the cleaning and fabrication process.

Under x-ray irradiation increasing the dose rate results in a positive shift in the curve. Generally, Poly-C shows the lowest photocurrent response among all the devices and an asymmetrical behaviour. This is due to the presence of defects introduced in the carbon-diamond interface (as described earlier). When irradiated these defects capture free electrons and holes (created by ionisation within the detector) leading to a build-up of space charge. This in turns creates an internal electric field opposing the external field leading a reduction in photo current. Due to the low photocurrent and high dark current of this device, a low signal to noise ratio (SNR) is observed. This result is of the same order as suggested in previous reports (SNR ranging from 10 to 100) by Fidanzio et.al. $[9,10]$ for a polycrystalline CVD diamond sample with thickness of $50 \mu \mathrm{m}$.

In contrast, the photocurrent plots in figure (4.b) and (4.c) of Poly-C/Ni and Poly-Pt show a similar behaviour (the induced current at a positive bias is approximately the same at a negative bias - within a factor of $10 \%$ ). It is believed that the addition of nickel to the carbon compensates for the effects of the defects within the carbon diamond interface due to its high conductivity. This is under further investigation. The amorphous carbon upon annealing is highly conductive. Again proof of this has been given in [12] Where excellent electrical properties were achieved in both pure carbon and Ni doped films through annealing at lower temperatures than the ones used for this work. The SNR of Poly-C/Ni and Poly-Pt is higher than 1000, satisfying the recommendations of the IAEA for medical dosimetry devices [14].

The significant increase in the current signal with increasing bias voltage can be interpreted as described in previous reports [15-18] where the higher the bias voltage, the greater the charge collection in the device and hence the rapid rate of e-h recombination phenomenon can be overcome. However the dark current increases.

All current measurements for the subsequent characterisation for the three devices were performed at $50 \mathrm{~V}$ bias voltages because it provided the best SNR whilst at the same time providing the optimum signal in terms of time response and stability.

\subsection{Signal amplitude}

Comparing the sensitivity parameters of the three devices there is a significant difference between the Poly-C, Poly-C/Ni and Poly-Pt devices. For the Poly-C device the sensitivity value was $\sim 6 \mathrm{nC} / \mathrm{Gy}$. This value is consistent with previous reports [9, 19] by Descamps et al and Fidanzio et al who found a sensitivity of (2 -3) nC/Gy for polycrystalline diamond samples. Their result is explained to be due to the high defect concentration in polycrystalline material. As for Poly-C/Ni and Poly-Pt the sensitivities were found to be 65 and $37 \mathrm{nC} / \mathrm{Gy}$, respectively. It should be noted that all sensitivity calculations were performed at $50 \mathrm{~V}$ bias.

For the three detectors reported here, specific sensitivities of about 3.8, 43.5 and $23.6\left(\mathrm{nC} \mathrm{Gy}^{-1} \mathrm{~mm}^{-3}\right)$, were obtained. The value of Poly-C/Ni is nearly the same order as typical values suggested in the literature (50- 135 $\left.\mathrm{nC} / \mathrm{Gy} . \mathrm{mm}^{3}\right)$ [9, 20-24] for PTW natural diamond and for polycrystalline CVD diamond $\left(\sim 77 \mathrm{nC} / \mathrm{Gy} \cdot \mathrm{mm}^{3}\right.$ at a bias of $100 \mathrm{~V})[10]$.

It should furthermore be noted that there is a significant difference in SNR for the three devices. In case of Poly-C a very low SNR value of $\sim 70-80$ was observed. This is due to the high dark current and its low photocurrent value. As for Poly-C/Ni and Poly-Pt the SNR is satisfactory as per the recommendations of the IAEA (report 374) [14] 
(higher than 1000) i.e. $\sim 3.3 \times 10^{3}$ and $\sim 10^{3}$, respectively. This is due to the very low dark current and relatively high photocurrent.

The measured value of the SNR, in Poly-C/Ni is of the same order as in previous reports by Galbiati et al. [11] who found a SNR of $3.3 \times 10^{4}$ for single crystal CVD diamond with DLC/Pt/Au contacts on both sides when exposed to Co-60 $\gamma$-rays ( $1 \mathrm{~Gy} / \mathrm{min}$ dose rate) at $100 \mathrm{~V}$. In that report the DLC was deposited by DC magnetron argon sputtering. In an other report [25] the SNR was 776 for single crystal diamond with a contact of nickel on one side and gold on the other side, which is consistent with the value of Poly-Pt.

Photoconductive gain or the charge collection efficiency (CCE), of an ionizing radiation detector, is considered one of the most important parameters that can determine the efficiency of the device in use for radiation detection. Generally, when the detector is irradiated, a current $\left(\mathrm{I}_{\text {generated }}\right)$ will be generated in the detector, which sequentially induces a current $\left(\mathrm{I}_{\text {measured }}\right)$ in the external circuit. The photoconductive gain value is known as the ratio between the current measured by the current meter and the theoretical current generated within the detector as a result of irradiation, i.e. dose rate. If the contacts allow charge injection it is given as the ratio between the average lifetime of the charge carriers, $\tau$, to the transit time $T_{r}\left(\right.$ Gain $\left.=\frac{I_{\text {measured }}}{I_{\text {generated }}}=\frac{\tau}{T_{r}}\right)$ of the dominating carrier type contributing to the signal.

The expected value of the theoretical current can be calculated from the equation:

$$
I_{\text {generated }}=\frac{D \rho e v}{w}
$$

Where: $D, \rho, e, v$ and $w$ are the dose rate, density of diamond, elementary charge, sensitive volume and the energy required to produce e-h pair in diamond (13.2 eV in natural diamond) respectively.

The calculated values are acquired at a bias voltage of $50 \mathrm{~V}$ and a dose rate of $1.5 \mathrm{~Gy} / \mathrm{min}$. The results are shown in table (2). It is obvious from table 2 that Poly-C has the lowest gain whilst Poly-C/Ni has the highest photoconductive gain. The gain value of Poly-C/Ni is approx. the same order to the typical value suggested by Fidanzio et al [9] ( 0.1) for polycrystalline diamond with dose rate and bias voltage of $3.5 \mathrm{~Gy} / \mathrm{min}$ and $100 \mathrm{~V}$, respectively.

\begin{tabular}{cc|cccccccc}
\hline Device & $\begin{array}{c}\text { Bias } \\
\text { voltage } \\
(\mathrm{V})\end{array}$ & $\begin{array}{c}\mathrm{I}_{\text {measured }} \\
(\mathrm{A})\end{array}$ & $\mathrm{SD}$ & $\begin{array}{c}\mathrm{I}_{\text {dark }} \\
(\mathrm{A})\end{array}$ & $\begin{array}{c}\mathrm{I}_{\text {generated }} \\
(\mathrm{A})\end{array}$ & $\begin{array}{c}\text { Gain } \\
\text { factor }\end{array}$ & $\begin{array}{c}\text { Sensitivity } \\
\mathrm{nC} / \mathrm{Gy}\end{array}$ & $\begin{array}{c}\text { Specific } \\
\text { Sensitivity } \\
\mathrm{nC} / \mathrm{Gy} \cdot \mathrm{mm}^{3}\end{array}$ & SNR \\
\hline Poly-C & 50 & $1.60 \times 10^{-10}$ & $7.40 \times 10^{-13}$ & $2.15 \times 10^{-12}$ & $6.5 \times 10^{-8}$ & $2.47 \times 10^{-3}$ & 6 & 3.8 & $70-80$ \\
Poly-C/Ni & 50 & $1.82 \times 10^{-9}$ & $4.84 \times 10^{-12}$ & $5.5 \times 10^{-13}$ & $1.04 \times 10^{-8}$ & $175 \times 10^{-3}$ & 65 & 43.5 & 3300 \\
Poly-Pt & 50 & $1.01 \times 10^{-9}$ & $3.77 \times 10^{-12}$ & $1 \times 10^{-12}$ & $4.16 \times 10^{-8}$ & $24.3 \times 10^{-3}$ & 37 & 23.6 & 1000 \\
\hline
\end{tabular}

Table (2): The gain factor, sensitivity, specific sensitivity and SNR of the three devices were obtained with irradiation by a dose rate of $1.5 \mathrm{~Gy} / \mathrm{min}$ and under bias voltage of $50 \mathrm{~V}$.

\subsection{Time response (Rise and fall-off times)}

The aim of this experiment is to measure the rise and fall-off times and the time dependency of the three different polycrystalline diamond samples. All current measurements were performed at a fixed dose rate and bias voltage of $1.5 \mathrm{~Gy} / \mathrm{min}$ and $50 \mathrm{~V}$, respectively. The rise time, $\tau_{10 \%-90 \%}$, is the time between $10 \%$ and $90 \%$ of the steady state current. The fall-off time is calculated by measuring the time between $90 \%$ and $10 \%$ of the steady state current. 

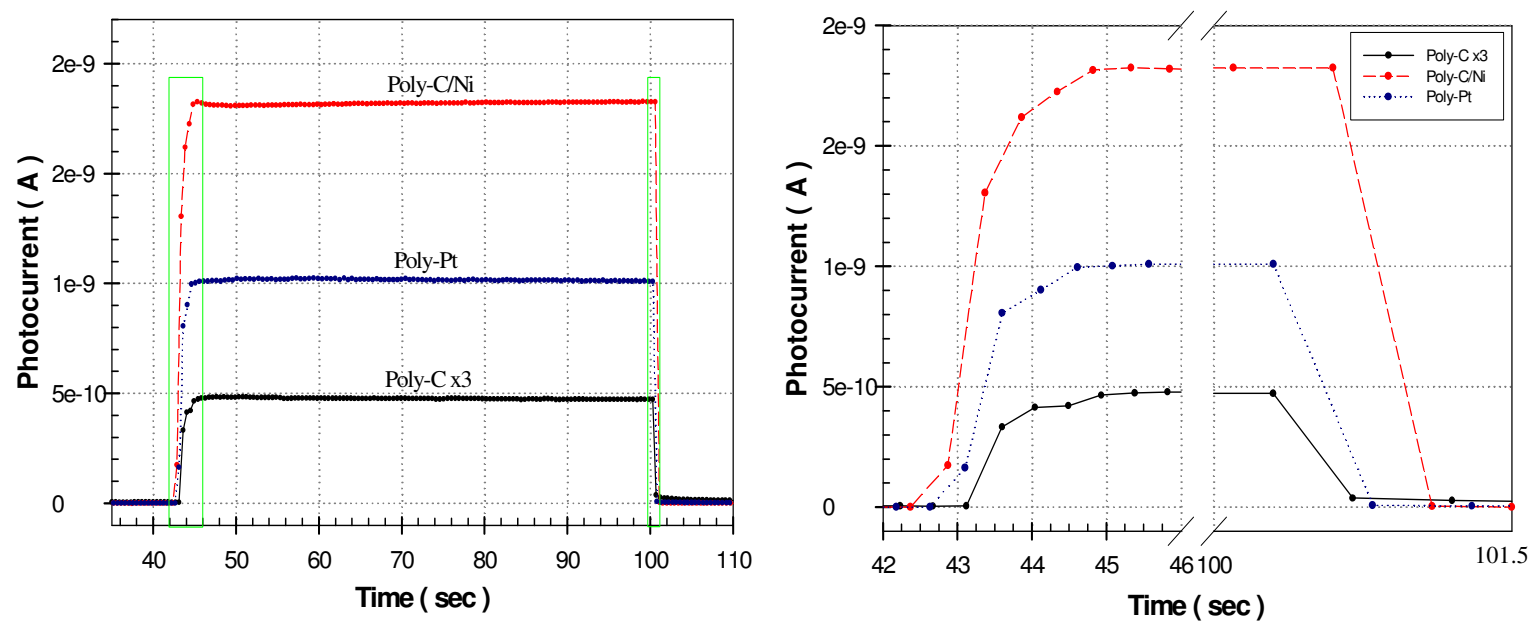

Figure (5): (a) Time response and (b) magnified rise and fall-off times of the three samples under fixed bias voltage and dose rate of $50 \mathrm{~V}$ and $1.5 \mathrm{~Gy} / \mathrm{min}$, respectively.

Figure (5) shows that the three devices (Poly-C, Poly-C/Ni and Poly-Pt) have fast rise and fall-off times. The rise times are approximately the same with 1.4, 1.1 and 1.25 seconds, respectively. The observed fall-off time is $\sim 0.4$ seconds and limited by the equipment. It must be noted that a fast detector response time is important as it leads to the ability to detect sudden changes in the x-ray beam.

Figure (6) displays the evolution of the photocurrent signal for the three polycrystalline diamond devices at a fixed bias of $50 \mathrm{~V}$ as a function of time.

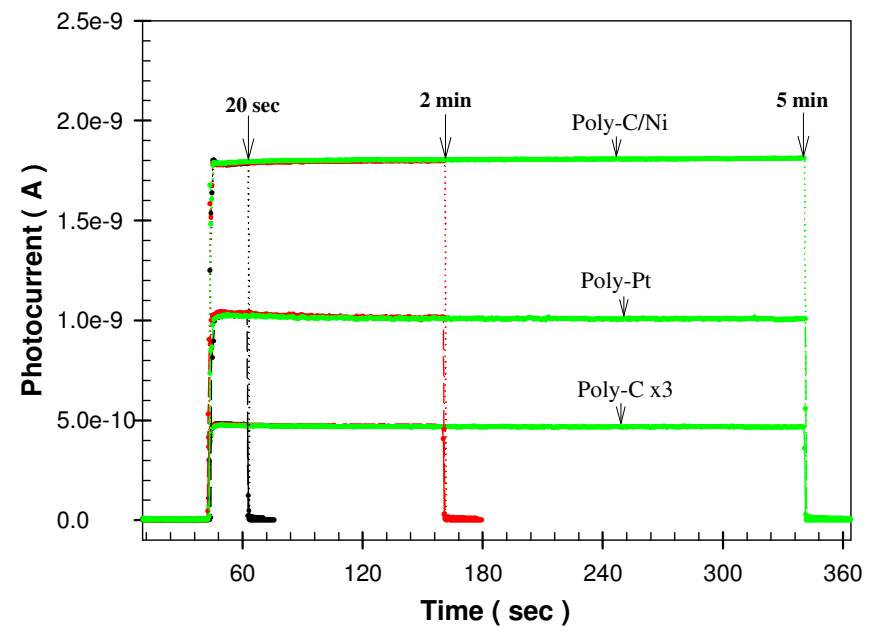

Figure (6): Time evolution of the three samples at different durations of x-ray switching on times of $20 \mathrm{sec}, 2$ and $5 \mathrm{~min}$. All current measurements were performed at a fixed dose rate of $1.5 \mathrm{~Gy} / \mathrm{min}$.

The current signals of the three samples are consistent, with in a tolerance of no more than $1.4 \%$, and share the same slope during the start of the x-ray irradiation. The variance in the photocurrent for the three samples could be reduced further if the internal electric field was given more time to stabilise. Additionally, the current signals show very fast rise and fall-off times. It should be noted that the previous observations that come from the three devices have an adequate value in both stability and reproducibility $(<0.5 \%)$. In another report, Buttar et al. [22] 
found that for polycrystalline CVD diamond the initial current response to x-ray irradiation increased by $\sim 20 \%$ with dose. This is believed to be due to the priming effect.

\subsection{Reproducibility}

Reproducibility (or repeatability) is considered one of the main parameters that should be fulfilled for use in dosimetric applications. It should be less than $0.5 \%$ as recommended by the International Atomic Energy Agency (IAEA) [14]. The stability and the reproducibility of the current response were estimated by taking the percentage ratio of the standard deviation (SD) to the average photocurrent value under exposure of a fixed test dose rate of $1.5 \mathrm{~Gy} / \mathrm{min}$ and $50 \mathrm{~V}$ bias voltages.

$$
\left(\text { Reproducibility }=\left[\left(\frac{S D}{\text { average }}\right) \%\right]\right)
$$

\begin{tabular}{cl|cc|c}
\hline \multirow{3}{*}{ Sample } & $\begin{array}{l}\text { No. of } \\
\text { re-use }\end{array}$ & $\begin{array}{c}\text { Average } \\
\text { current } \\
(\mathrm{A})\end{array}$ & SD & Reproducibility \% \\
\hline \multirow{3}{*}{ Poly-C } & $1^{\text {st }}$ & $1.6 \mathrm{e}-10$ & $7.36 \mathrm{e}-13$ & 0.459 \\
& $2^{\text {nd }}$ & $1.59 \mathrm{e}-10$ & $7.40 \mathrm{e}-13$ & 0.464 \\
& $3^{\text {rd }}$ & $1.61 \mathrm{e}-10$ & $7.43 \mathrm{e}-13$ & 0.459 \\
& $\Sigma$ & $1.60 \mathrm{e}-10$ & $7.40 \mathrm{e}-13$ & $\mathbf{0 . 4 6 1}$ \\
\hline \multirow{3}{*}{ Poly-C/Ni } & $1^{\text {st }}$ & $1.81 \mathrm{e}-9$ & $4.22 \mathrm{e}-12$ & 0.233 \\
& $2^{\text {nd }}$ & $1.82 \mathrm{e}-9$ & $4.07 \mathrm{e}-12$ & 0.223 \\
& $3^{\text {rd }}$ & $1.83 \mathrm{e}-9$ & $6.23 \mathrm{e}-12$ & 0.341 \\
& $\Sigma$ & $1.82 \mathrm{e}-9$ & $4.84 \mathrm{e}-12$ & $\mathbf{0 . 2 6 6}$ \\
\hline \multirow{3}{*}{ Poly-Pt } & $1^{\text {st }}$ & $1.01 \mathrm{e}-9$ & $3.62 \mathrm{e}-12$ & 0.358 \\
& $2^{\text {nd }}$ & $1.01 \mathrm{e}-9$ & $3.40 \mathrm{e}-12$ & 0.336 \\
& $3^{\text {rd }}$ & $1.01 \mathrm{e}-9$ & $4.30 \mathrm{e}-12$ & 0.425 \\
& $\Sigma$ & $1.01 \mathrm{e}-9$ & $3.77 \mathrm{e}-12$ & $\mathbf{0 . 3 7 3}$ \\
\hline
\end{tabular}

Table (3) Reproducibility of the three polycrystalline diamond samples under fixed bias voltage and dose rate of $50 \mathrm{~V}$ and $1.5 \mathrm{~Gy} / \mathrm{min}$, respectively.

According to the data provided in table (3) reasonable reproducibility $(<0.5 \%)$ is obtained, which it satisfies the recommended limit of the IAEA for dosimetric assessments. It demonstrates the high stability of the steady state current. The values are consistent with typical values reported in previous work $(0.32 \%)$ by Galbiati et al. [11] for a single crystal diamond having a DLC/Pt/Au contact exposed to irradiation of Co-60 $\gamma$-rays at an electric field of $0.2 \mathrm{~V} / \mu \mathrm{m}$. They are also consistent with those reported by Fidanzio et.al, [10] who found a reproducibility of $\sim 0.5 \%$ for polycrystalline CVD diamond after pre-irradiation with $5 \mathrm{~Gy}$.

\subsection{Linearity and dose rate dependence}

The aim of this section is to study the linearity of the photocurrent as a function of the irradiated dose rates. The induced current signals are obtained at different dose rates ranging from 0.155 to 2.45 (Gy/min) at fixed bias voltages of $50 \mathrm{~V}$. The three devices were pre-irradiated with an x-ray dose of $\sim 10$ Gy before any measurements were taken. It should be noted that minimal difference is observed before and after the pre-irradiation dose was performed.

Generally, figure (7.a) shows that the majority of the signals in the three samples display a step-like change in current when irradiated with x-rays without observing the undesired effects such as priming or the persistent photocurrent. In addition there is an increase in the photocurrent as the dose rate increases but not at the same rate as can be seen in figure (7.b). It should be noted that the time difference between $90 \%$ and $100 \%$ of the steady state (equilibrium) current is negligible. An exception to the last observation is Poly-C, which displays a 
stabilization time between $90 \%$ and $100 \%$ of the steady state current which is slightly higher than that of the Poly$\mathrm{C} / \mathrm{Ni}$ and Poly-Pt samples but only at low dose rates.

Figure (7.a) shows that the Poly-C sample has the lowest photocurrent response among the samples, while for Poly-C/Ni the photocurrent has the highest response with a high stability in the steady state current. Poly-Pt figure (7.a) shows a small overshoot at the beginning of the x-ray irradiation followed by a stable steady state current.

The linearity of a radiation detector, i.e. the variation of the current $I$ as a function of the irradiated dose rate $(D)$, can be expressed using the Fowler relationship [26]:

$$
I=I_{\text {dark }}+R D^{\Delta}
$$

Where: $I_{\text {dark }}$ is the dark current. The exponent $\Delta$, sometimes referred to as the fitting coefficient, or the linearity index is a constant showing the deviation from linearity. It usually varies between 0.5 and 1.0 if all the traps in the crystal have the same capture cross section. The measured value of $\Delta$ is expected to equal one if all the traps are distributed uniformly or quasi uniformly inside the diamond material. $\Delta$ equals approximately 0.5 if free charge carrier recombination dominates i.e. in the case of very low trap densities or high excitation rates. Additionally, the value of $\Delta$ can exceed one, if the traps in the crystal have different capture cross sections or are distributed non-uniformly.
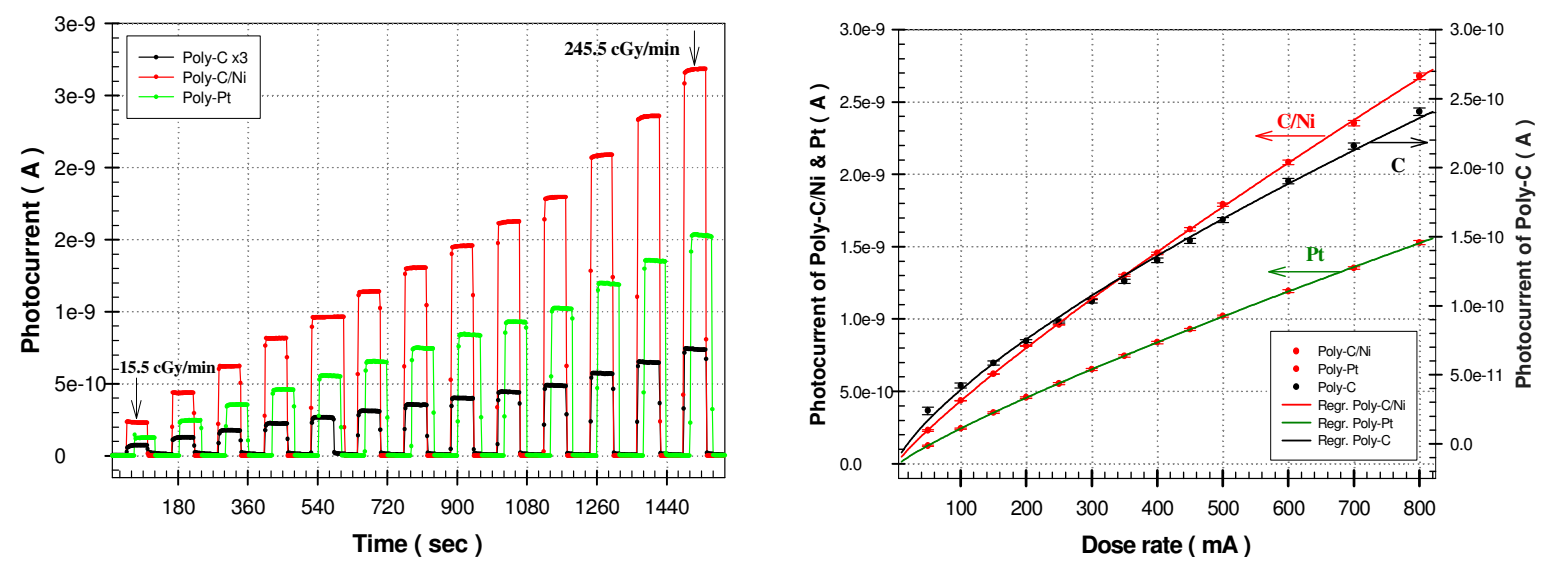

Figure (7): (a) Current signals as a function of time and (b) dose rate dependence in the three samples at different dose rates ranging from 15.5 to $245.5 \mathrm{cGy} / \mathrm{min}$. The measurements were performed under $50 \mathrm{~V}$ bias voltage.

Deviation from linearity can also be attributed to charge carriers trapped in defect levels (trapping centres), which reduce the charge lifetimes $(\tau)$ [27]. Measuring $\Delta$ is particularly important in the case of x-ray dosimetry, because using that value allows one to measure the corrective factor that can allow for calculating absorbed dose. In the case of $\Delta$ equals one, the integrated photocurrent of the device does not depend on the dose rate, which is an advantage in dosimetry applications.

Figure (7.b) displays the photocurrent versus dose rate of the three devices (Poly-C (blue), Poly-C/Ni (black) and Poly-Pt (red)). The photocurrent values were extracted from figure (7.a). As seen in both Poly-C/Ni and Poly-Pt a relationship is observed between the current and the dose rate with similar $\Delta$ values of 0.86 and 0.85 , respectively. These values indicate that there is an approx. uniform trap distribution which could be related to the structure of polycrystalline diamond. The measured values of $\Delta$, in both Poly-C/Ni and Poly-Pt have approx. the same order to the typical values suggested in the literature (0.86-1.035) [9, 20-24] for both PTW natural diamond and synthetic CVD diamond devices. A similar result was reported by Buttar et al. [22] for polycrystalline CVD diamond dosimeters with $\mathrm{Cr} / \mathrm{Au}$ contact on both sides using $250 \mathrm{kV}$ X-rays, where a slightly non-linear relationship with dose rate $(\Delta \approx 0.91)$ was observed. 
However for Poly-C the resulting "best" fit line seems to indicate a power relationship with a $\Delta$ value of 0.73 . One possible explanation for this reduced $\Delta$ value could be the carbon deposition creating defects near the carbondiamond interface. This in turn could lead to a higher accumulation of negative charge in the region close to the anode and positive charge near the cathode, which in turn reduces the external applied electric field, and consequently the current response. The increase in space charge within this sample may be due to the deposited amorphous carbon producing a higher defect concentration.

A very low $\Delta$ (non linear) value of 0.7 was observed for a polycrystalline CVD diamond sample in a previous report; the sub-linear relationship is attributed to the reduced charge collection efficiency of the sample with dose rate. This effect is attributed to the presence of traps which effects the dose rate dependence [10]. The higher the dose rates, the higher the e-h creation within the device leading to a higher probability that the traps within the detector will be filled. This build-up of space charge reduces the external electric field, consequently reducing the response of the device. This could be the reason for the non-linearity at this case.

It should be noted that the value of $\Delta$ could approach 1 at a high bias voltages as it would increase the charge collection efficiency (a higher proportion of the produced charge carriers are collected at the external circuit) of the device. However this would be at the expense of the SNR due to the increase in the dark current with bias.

\section{Conclusions}

The purpose of this study is to develop x-ray dosimeters which are as close to tissue equivalent as possible using PLD to deposit Carbon electrodes on polycrystalline diamond. In both Poly-C/Ni and Poly-Pt the dark currents are very low (below a few pA between $-200 \mathrm{~V}$ and $+200 \mathrm{~V}$ ). Poly-C shows a relatively high dark current, however this is still below $25 \mathrm{pA}$ at the same bias interval.

Poly-C shows the lowest photocurrent response among all the devices (i.e. the current response of the three devices are $1.6 \times 10^{-10}, 1.8 \times 10^{-9}$ and $1.01 \times 10^{-9} \mathrm{~A}$, respectively). The majority of the signals in the three devices display a step-like change in current when irradiated with $\mathrm{x}$-rays without observing undesired effects such as the persistent photocurrent.

In both Poly-C/Ni and Poly-Pt an approximately linear relationship is observed between the current and the dose rate with $\Delta$ values of 0.86 and 0.85 , respectively. For Poly-C the resulting "best" fit line seems to indicate a power relationship with a lower $\Delta$ value of 0.73 . Comparing the sensitivity parameters of the three devices there is a significant difference between the Poly-C, Poly-C/Ni and Poly-Pt devices (i.e. 6, 65 and 37 nC/Gy, respectively). Reasonable reproducibility $(<0.5 \%)$ is observed for the three devices (i.e. $0.46,0.26$ and $0.37 \%$, respectively) which satisfy the recommendations of the IAEA

In case of Poly-C a very low SNR value of $\sim 70-80$ was observed. As for Poly-C/Ni and Poly-Pt the SNR is satisfactory as per the recommendations of the IAEA (higher than 1000) i.e. $\sim 3300$ and $\sim 1000$, respectively. The rise time of the three devices have approximately the same values of 1.4, 1.1 and 1.25 seconds, respectively. Also, the fall-off times of the three devices are less than $\sim 0.4$ second.

The obtained results confirmed that the contact deposition of C/Ni by PLD technique on polycrystalline diamond was comparable or even better than contacts fabricated by conventional sputtering of Pt, as the novel device "Poly-C/Ni" successfully satisfied the majority of the dosimetric requirements set out within the international standards. 


\section{Acknowledgments}

I would like to thank Y. Y. Tan's in the Advanced Technology Institute and the members of the University of Surrey's Radiation Detection Research Group especially S. Chaudhuri and P. Bryant for their help and assistance during the preparation of this paper.

\section{$5 \quad$ References}

1. Bergonzo, P., Brambilla, A., Tromson, D., Mer, C., Guizard, B., Marshall, R. D., Foulon, F., CVD diamond for nuclear detection applications. Nuclear Instruments and Methods in Physics Research Section A: Accelerators, Spectrometers, Detectors and Associated Equipment, 2002. 476(3): p. 694-700.

2. Robertson, J., Mechanical properties and coordinations of amorphous carbons. Physical Review Letters, 1992. 68(2): p. 220.

3. Gutiérrez, A., Díaz, J., López, M. F., X-ray absorption spectroscopy study of pulsed-laser-evaporated amorphous carbon films. Applied Physics A: Material Science \& Processing, 1995. 61(2): p. 111-114.

4. Diaz, J., Gago, J. A. Martin, Ferrer, S., Comin, F., Abello, L., Lucazeau, G., Raman spectroscopy of carbon films grown by pulsed laser evaporation of graphite. Diamond and Related Materials, 1992. 1(7): p. 824-827.

5. Almaviva, S., Marinelli, M., Milani, E., Prestopino, G., Tucciarone, A., Verona, C., Verona-Rinati, G., Angelone, M., Pillon, M., Extreme UV photodetectors based on CVD single crystal diamond in a p-type/intrinsic/metal configuration. Diamond and Related Materials, 2009. 18(1): p. 101-105.

6. Kuzmany, H., Pfeiffer, R., Salk, N., Günther, B., The mystery of the $1140 \mathrm{~cm}-1$ Raman line in nanocrystalline diamond films. Carbon, 2004. 42(5-6): p. 911-917.

7. Robertson, A.C.F.a.J., Resonant Raman spectroscopy of disordered, amorphous, and diamondlike carbon. PHYSICAL REVIEW B, 2001. 64, 075414.

8. Di Benedetto, R., Marinelli, Marco, Messina, G., Milani, E., Pace, E., Paoletti, A., Pini, A., Santangelo, S., Scuderi, S., Tucciarone, A., Verona-Rinati, G., Bonanno, G., Influence of metal-diamond interfaces on the response of UV photoconductors. Diamond and Related Materials, 2001. 10(3-7): p. 698-705.

9. Fidanzio, A., Azario, L., Viola, P., Ascarelli, P., Cappelli, E., Conte, G., Piermattei, A., Photon and electron beam dosimetry with a CVD diamond detector. Nuclear Instruments and Methods in Physics Research Section A: Accelerators, Spectrometers, Detectors and Associated Equipment, 2004. 524(1-3): p. 115-123.

10. Fidanzio, A., Azario, L., Venanzi, C., Pinzari, F., Piermattei, A., Production and testing of a synthetic diamond film radiation dosimeter for radiotherapy. Nuclear Instruments and Methods in Physics Research Section A: Accelerators, Spectrometers, Detectors and Associated Equipment, 2002. 479(2-3): p. 661-667.

11. Galbiati, A., et al., Performance of Monocrystalline Diamond Radiation Detectors Fabricated Using TiW, Cr/Au and a Novel Ohmic DLC/Pt/Au Electrical Contact. IEEE Transactions on Nuclear Science, 2009. 56(4): p. 1863-1874.

12. Jayawardena, K.D.G.I., Tan, Y. Y., Fryar, J., Shiozawa, H., Silva, S. R. P., Henley, S. J., Fuge, G. M., Truscott, B. S., Ashfold, M. N. R., Highly conductive nanoclustered carbon:nickel films grown by pulsed laser deposition. Carbon, 2011. 49(12): p. 3781-3788.

13. Miyajima, Y.H., S. J.; Adamopoulos, G.; Stolojan, V.; Garcia-Caurel, E.; Drévillon, B.; Shannon, J. M.; Silva, S. R. P., Pulsed laser deposited tetrahedral amorphous carbon with high sp3 fractions and low optical bandgaps. Journal of Applied Physics, 2009. 105(7): p. 073521-073521-8.

14. Agency, I.A.E., Calibration of dosimeters used in radiotherapy in Technical reports series -- no. 374. 1994: Vienna.

15. Iwakaji, Y., Kanasugi, M., Maida, O., Takeda, Y., Saitoh, Y., Ito, T., Characterization of soft-X-ray detectors fabricated with high-quality CVD diamond thin films. Applied Surface Science, 2008. 254(19): p. 6277-6280.

16. Matsubara, H., Saitoh, Y., Maida, O., Teraji, T., Kobayashi, K., Ito, T., High-performance diamond soft-X-ray detectors with internal amplification function. Diamond and Related Materials, 2007. 16(4-7): p. 1044-1048.

17. Pauling, L., Electronic Processes in Ionic Crystals. By N. F. Mott and R. W. Gurney. The Journal of Physical Chemistry, 1941. 45(7): p. 1142-1142.

18. Boer, K.W., Survey of Semiconductor Physics. Barriers, Junctions, Surfaces and devices. Vol. vol. II. 1992, New York: Van Norstrand Reinhold.

19. Descamps, C., Tromson, D., Tranchant, N., Isambert, A., Bridier, A., De Angelis, C., Onori, S., Bucciolini, M., Bergonzo, P., Clinical studies of optimised single crystal and polycrystalline diamonds for radiotherapy dosimetry. Radiation Measurements, 2008. 43(2-6): p. 933-938.

20. Lansley, S.P., Betzel, G.T., Baluti, F., Reinisch, L., Meyer, J., CVD Diamond X-ray Detectors for Radiotherapy Dosimetry. Christchurch, New Zealand: 8th Annual IEEE Conference on Sensors, 25-28 Oct 2009. Proceedings of 8th Annual IEEE Conference on Sensors, 2009. 
21. Bucciolini, M., Borchi, E., Bruzzi, M., Casati, M., Cirrone, P., Cuttone, G., De Angelis, C., Lovik, I., Onori, S., Raffaele, L., Sciortino, S., Diamond dosimetry: Outcomes of the CANDIDO and CONRAD INFN projects. Nuclear Instruments and Methods in Physics Research Section A: Accelerators, Spectrometers, Detectors and Associated Equipment, 2005. 552(1-2): p. 189-196.

22. Buttar, C.M., Airey, R., Conway, J., Hill, G., Ramkumar, S., Scarsbrook, G., Sussmann, R. S., Walker, S., Whitehead, A., A study of radiotherapy dosimeters based on diamond grown by chemical vapour deposition. Diamond and Related Materials, 2000. 9(3-6): p. 965-969.

23. Cirrone, G.A.P., Cuttone, G., Lo Nigro, S., Mongelli, V., Raffaele, L., Sabini, M. G., Dosimetric characterization of CVD diamonds in photon, electron and proton beams. Nuclear Physics B - Proceedings Supplements, 2006. 150: p. 330-333.

24. Górka, B., Nilsson, B., Svensson, R., Brahme, A., Ascarelli, P., Trucchi, D. M., Conte, G., Kalish, R., Design and characterization of a tissue-equivalent CVD-diamond detector for clinical dosimetry in high-energy photon beams. Physica Medica, 2008. 24(3): p. 159-168.

25. Tranchant, N., Tromson, D., Descamps, C., Isambert, A., Hamrita, H., Bergonzo, P., Nesladek, M., High mobility single crystal diamond detectors for dosimetry: Application to radiotherapy. Diamond and Related Materials, 2008. 17(7-10): p. 1297-1301.

26. Fowler, J.F., Solid state electrical conduction dosimeters. ,edited by Attix, Roesch, and Tochlin, Radiation Dosimetry II (Academic Press Inc., 1966), Chapter 14, p. 308.

27. Ramkumar, S., Buttar, C. M., Conway, J., Whitehead, A. J., Sussman, R. S., Hill, G., Walker, S., An assessment of radiotherapy dosimeters based on CVD grown diamond. Nuclear Instruments and Methods in Physics Research Section A: Accelerators, Spectrometers, Detectors and Associated Equipment, 2001. 460(2-3): p. 401-411. 